\title{
Dietary carbohydrates and management of the gut environment of pigs.
}

Bach Knudsen, K. E.; Lærke, H. N.; Hedemann, Mette Skou; Jensen, B. B.; Hansen, Christian Fink

Published in:

The British Society of Animal Science

Publication date:

2003

Document version

Early version, also known as pre-print

Citation for published version (APA):

Bach Knudsen, K. E., Lærke, H. N., Hedemann, M. S., Jensen, B. B., \& Hansen, C. F. (2003). Dietary carbohydrates and management of the gut environment of pigs. In The British Society of Animal Science (pp. 231) http://www.bsas.org.uk/wp-content/themes/bsas/proceedings/Pdf2003/cover03.pdf 


\section{Dietary carbohydrates and management of the gut environment of pigs}

K. E. Bach Knudsen, H. N. Lærke, M. S. Hedemann, B. B. Jensen and C. F. Hansen

Danish Institute of Agricultural Sciences, Dept. Animal Nutrition and Physiology, PO Box 50, DK-8830 Tjele Denmark. E-mail: knuderik.bachknudsen@agrsci.dk.

Introduction Dietary carbohydrates constitute a major fraction of the diets for pigs. The carbohydrate fraction consists of mono-, di- and oligosaccharides and two broad classes of polysaccharides - starch and non-starch polysaccharides (NSP). The carbohydrate fraction has a diverse composition in terms of constituent sugars (pentoses, hexoses, deoxysugars, etc.), glycosidic linkages (alfa or beta), size (degree of polymerisation from one to several thousand), and physical form (soluble in water, insoluble, cation and adsorbing properties). It is now evidential clear that the composition of the carbohydrate fraction influences the digestion and absorption processes of carbohydrates and other nutrients in the various parts of the gastrointestinal tract, it has a profound influence on the secretory response of the gut to feed intake, the volume flow, the mucosal architecture, the composition of the gut flora and the development of the gastrointestinal tract.

Oligosaccharides The intestinal mucosa of the pig lacks the enzymes capable of cleaving a number of oligosaccharides that are naturally present in plant materials (i.e. raffinose-oligosaccharides, fructooligosaccharides) or used as feed additives (i.e. neosugar, transgalactooligosaccharides). Collectively, these oligosaccharides are referred to as nondigestible oligosaccharides (NDO). NDO were earlier considered as an antinutritional factor, which could potentially accumulate in the small intestine, cause osmotic diarrhoea, and, because of their rapid fermentation and high gas production, cause discomfort for the animals. Recently, there has been a growing interest in NDO because of their possible prebiotic properties, i.e. stimulation of the growth and (or) activity of one or a limited number of desired bacteria in colon and thus, exclude the pathogens. In man, it has been demonstrated that inulin and oligofructose independent on chain length have significant prebiotic properties by selective stimulating of the growth of bifidobacteria in the colon. The results from studies with pigs, however, are less convincing and it seems as if the concept, because of economically constraints, can be difficult to use for older pigs.

Starch is the most abundant carbohydrate in diets for pigs. Starch is a mixture of the linear $\alpha(1-4)-$ linked amylose and the branched $\alpha(1-4),(1-6)$ - linked amylopectin. Most of the ingested starch is efficiently broken down by the combination of secreted $\alpha$-amylase and enzymes located on the intestinal surface membrane. A variable fraction of starch (resistant starch, RS), however, will be resistant against degradation in the small intestine and may have properties similar to NSP. A high amylose cornstarch diet fed together with Bifidobacterium longum has been shown to increase the number of bifidobacteria in the faeces compared to a low amylose cornstarch diet. Studies with catheterised pigs also show that high levels of RS change the composition of short-chain fatty acids produced towards more butyrate.

NSP are the main carbohydrate fraction not digested by enzymes in the small intestine of pigs. At this site of the gastrointestinal tract, NSP composition (type of polysaccharides, solubility) will have a major influence on the physicochemical properties of the digesta materials. However, because of the microbial colonisation of the stomach and small intestine, some disappearance $(\sim 24 \%)$ of NSP occurs in the upper intestinal tract. Results obtained with cereal diets consistently show a higher degradation of the linear and relatively soluble $\beta$-glucan compared to the branchedchain arabinoxylans from wheat, rye and oats. The molecular weight of $\beta$-glucan was reduced up to 20 -fold in the upper gastrointestinal tract but with relatively low digestibility until the terminal ileum. The amount of dry matter that passes from the small to the large intestine is related to dietary fibre intake and with NSP representing in the order of $35-45 \%$ of the undigested residue. The degradation of the NSP polymers in the large intestine is influenced by factors such as solubility, crystallinity and degree of lignification. NSP have a strong influence on the activity and composition of the commensal microflora, the composition of the short-chain fatty acids produced but it does not seem to have a selective influence on specific strains of microorganisms as has been identified for some NDO (i.e. various fructans). Exogenous enzymes will degrade parts of NSP to mono-, di- and oligomers starting already in the small intestine. In this way the NSP may potentially be used as a substrate to produce oligomers with prebiotic properties. However, convincing in vivo data are still lacking.

The feed structure is tightly connected with the feed composition and way the feed is processed. In particular, the fibre content of the raw material plays a role; for example barley produces a more coarse meal than wheat when milled to pass the same screen size. Feeding a coarse diet results in a digesta material that is more coherent, which in turn influences the digestive processes, the microbial ecosystem and the morphology of the gastrointestinal tract. However, the down side of this concept is that, as a result of the coherent properties of coarsely-ground materials, there will be a bigger risk of encapsulation of nutrients, which are then not available for digestion by enzymes in the small intestine.

Conclusions The diverse nature of feed carbohydrates makes it possible to influence the gut environment of the pig by choosing among the various feedstuffs and processing conditions. 